\title{
NIVELES DE LISINA Y TREONINA DIGESTIBLE EN DIETAS SORGO-PASTA DE SOYA PARA CERDOS EN CRECIMIENTO
}

\author{
DIGESTIBLE LYSINE AND THREONINE LEVELS IN SORGHUM-SOYBEAN MEAL DIETS \\ FOR GROWING PIGS
}

\author{
López, M. ${ }^{1}$, Figueroa, J.L. ${ }^{2 *}$, González, M.J. ${ }^{1}$, Miranda, L.A. ${ }^{1}$, Zamora, V. ${ }^{2}$ y Cordero, J.L. ${ }^{2}$ \\ ${ }^{1}$ Departamento de Zootecnia. Universidad Autónoma Chapingo. km 38.5. Carretera México-Texcoco. \\ Chapingo. CP 56230. Estado de México. \\ ${ }^{2}$ Programa de Ganadería. Campus Montecillo. Colegio de Postgraduados. km 36.5. Carretera México- \\ Texcoco. Montecillo. Texcoco. CP 56230. Estado de México. *jlfigueroa@colpos.mx
}

\section{Palabras clave adicionales}

Aminoácidos esenciales. Comportamiento productivo. Características de canal.

\section{RESUMEN}

Se evaluó el efecto de diferentes niveles de lisina y treonina en dietas sorgo-pasta de soya para cerdos en crecimiento, sobre el comportamiento productivo, las carácterísticas de la canal y la concentración de nitrógeno de la urea plasmática (NUP). En el experimento 1 se evaluaron cinco niveles de lisina digestible $(0,67 ; 0,75$ 0,$83 ; 0,91$ y $0,99 \%$ ) durante cuatro semanas. Se utilizaron 50 cerdos machos castrados con peso promedio inicial de $22,34 \pm 3,45 \mathrm{~kg}$. En el experimento 2 se evaluaron cinco niveles de treonina digestible $(0,42 ; 0,47 ; 0,52 ; 0,57$ y $0,62 \%)$ durante seis semanas. Se utilizaron 50 cerdos $(25$ machos castrados y 25 hembras) con peso promedio inicial de $24,02 \pm 3,73 \mathrm{~kg}$. Los animales fueron distribuidos en un diseño experimental completamente al azar con cinco repeticiones y dos cerdos por unidad experimental. Las variables de respuesta evaluadas en ambos experimentos fueron: consumo diario de alimento (CDA), ganancia diaria de peso (GDP), conversión alimenticia (CA), ganancia de carne magra (GCM), peso final (PF), grasa dorsal (GD), porcentaje de carne magra (\%CM), área del músculo longissimus (AML) y NUP. Las variables medidas semanalmente (CDA, GDP y CA), fueron analizadas con PROC MIXED de SAS; las otras variables fueron analizadas con PROC GLM. La comparación de medias se realizó con la prueba de Tukey, y se utilizaron contrastes ortogonales para detectar efectos lineales,

Recibido: 2-4-08. Aceptado: 22-10-08.

\section{AdDitional KEYWORDS}

Essential amino acids. Growth performance. Carcass characteristics.

cuadráticos y cúbicos. El mejor nivel de lisina digestible para CDA y GDP fue $0,75 \%$; para CA fue $0,91 \%$, y para PF fue de $0,99 \%$. El análisis de contrastes mostró efecto cúbico del nivel de lisina sobre CDA, lineal para CA y cuadrático para AML, \%CM y NUP. Los niveles de treonina digestible no afectaron las variables de respuesta productiva, ni las características de la canal; sólo se observó una reducción lineal del NUP al aumentar la treonina digestible. Los machos castrados también redujeron el NUP al aumentar el nivel de treonina digestible en la dieta. Estos resultados indican que el nivel de lisina digestible afecta de manera diferente las variables productivas, las características de la canal, y la concentración de nitrógeno uréico en plasma; y que el nivel de treonina digestible no afecta las variables analizadas aunque se reduce el NUP en cerdos en crecimiento al aumentar la treonina digestible en la dieta.

\section{SUMMARY}

Was evaluated the effect of digestible lysine and threonine supplemented to sorghum-soybean meal diets for growing pigs on growth performance, carcass characteristics, and plasma urea nitrogen concentration (NUP). In experiment 1 , five dietary digestible lysine levels $(0.67,0.75,0.83$, 0.91 and $0.99 \%$ ) were evaluated during four weeks. Fifty barrows with $22.34 \pm 3.45 \mathrm{~kg}$ of initial 


\section{LÓPEZ, FIGUEROA, GONZÁLEZ, MIRANDA, ZAMORA Y CORDERO}

weight were used. In experiment 2 five dietary digestible threonine levels $(0.42,0.47,0.52,0.57$ and $0.62 \%$ ) were evaluated during six weeks. Fifty pigs ( 25 barrows and 25 gilts) with $24.02 \pm$ $3.73 \mathrm{~kg}$ of initial weight were used. Pigs were alloted in a completely randomized design with five replicates of two pigs per experimental unit. The analyzed variables were as follows: average daily feed intake (CDA), average daily gain (GDP), feed:gain ratio (CA), fat free lean gain (GCM), final body weight (PF), backfat thickness (GD), lean meat percentage $(\% \mathrm{CM})$, longissimus muscle area (AML), and plasma urea nitrogen concentration (NUP). The CDA, GDP and CA were recorded weekly and analyzed with the PROC MIXED procedure of SAS; the variables obtained at the end of the experiment were analyzed with GLM procedure. The means comparison was performed with Tukey test; and orthogonal contrasts were applied to detect linear, quadratic or cubic effects. The best digestible lysine level for CDA and GDP was $0.75 \%$; for CA was $0.91 \%$; and for PF was $0.99 \%$. The orthogonal contrasts analysis showed a cubic effect of digestible lysine on CDA; a lineal effect on CA; and a quadratic effect on AML, \%CM, and NUP. The digestible threonine levels did not affect growth performance, nor carcass characteristics; they just linearly reduced NUP as digestible threonine levels increased. The barrows also reduced NUP as the level of dietary digestible threonine increased. These results indicate that the level of digestible lysine affects differently growth performance, carcass characteristics and plasma urea nitrogen concentration; and that digestible threonine levels did not affect the analyzed variables.

\section{INTRODUCCIÓN}

Los costos de alimentación en una explotación porcina son casi siempre mayores del $70 \%$, lo que limita las utilidades del productor. Por eso, es importante buscar alternativas que permitan optimizar los recursos, como la adición de aminoácidos (AA) sintéticos ya que permite reducir el contenido de proteína bruta $(\mathrm{PB})$ en la ración y posibilita satisfacer las necesidades de AA esenciales limitantes, e incrementa la eficiencia alimenticia (Araki, 1990), lo que repercute en un beneficio económico para el productor.

La lisina y treonina son el primero y segundo AA limitantes en dietas a base de sorgo y maíz y pasta de soya para cerdos (Fontes et al., 2000), y treonina puede ser el primer AA limitante cuando se agrega solamente lisina sintética a la dieta, sin considerar los otros AA (Saldana et al., 1994) en la formulación del alimento. La adición de AA sintéticos puede resolver el problema de la deficiencia de unos y el exceso de otros AA (Hansen et al., 1993); además, disminuye la excreción del nitrógeno y la contaminación ambiental (NRC, 1998). Además se puede mejorar el comportamiento productivo, por efecto del nivel de lisina en la dieta, tanto en el consumo de alimento, (Cline et al., 2000); como en la ganancia de peso (Ferreira et al., 2005). También se ha encontrado que al aumentar el nivel de lisina en la dieta se incrementa el depósito de proteína en la canal de cerdos en crecimiento (Fontes et $a l ., 2000)$ y una respuesta lineal sobre el músculo longissimus (Hahn et al.,1995). Igualmente el aumento de la treonina afecta a la conversión alimenticia en cerdos en crecimiento y finalización (Ettle et al., 2004) y puede mejorarse la ganancia de peso (Pichardo et al., 2003).

El requerimiento de lisina y treonina para cerdos puede ser monitoreado por la concentración de urea en plasma, pues es la forma de transporte del exceso de nitrógeno hacia el riñón para su excreción (Wei y Zimmerman, 1998). La reducción de urea presumiblemente refleja un uso más eficiente del nitrógeno total, por ello, los niveles más bajos de urea en cerdos pudieran indicar los niveles y el balance adecuados de AA, debido a que hay una mayor retención de nitrógeno (Coma et al.,1995). El objetivo del presente trabajo fue determinar el mejor nivel de lisina y treonina digestibles para el comportamiento productivo, las características de la canal y la concentración de urea en plasma de cerdos en crecimiento alimentados con dietas sorgo-pasta de soya. 


\section{LISINA Y TREONINA DIGESTIBLE EN DIETAS PARA CERDOS EN CRECIMIENTO}

\section{MATERIAL Y MÉTODOS}

Esta investigación se llevó a cabo en la Granja Experimental de la Universidad Autónoma Chapingo, ubicada en Texcoco, Estado de México, a una altitud de 2250 msnm. El clima es templado subhúmedo con lluvias en verano, con temperatura media anual de $15,2^{\circ} \mathrm{C}$ y precipitación media anual de 644,8 mm (García, 1988). Según la estación metereológica de la Universidad Autónoma Chapingo, la temperatura promedio, mínima y máxima a la intemperie fueron 18,5 ; $-1,5$ y 26,$4 ;$ y 19,$0 ; 0,2$, y $26,9^{\circ} \mathrm{C}$ para 1 os experimentos 1 y 2 , respectivamente.

\section{TRATAMIENTOS}

Experimento 1: Se utilizaron cinco niveles de lisina digestible en la dieta $(0,67 ; 0,75$; 0,$83 ; 0,91$ y $0,99 \%$ ) para cerdos en crecimiento. El valor $0,83 \%$ de lisina digestible es el recomendado por el NRC (1998). Se utilizaron 50 cerdos machos castrados híbridos (Pietrain $\times$ Landrace $\times$ Yorkshire), con un peso promedio inicial de $22,34 \pm 3,45 \mathrm{~kg}$, distribuidos al azar en cinco tratamientos, utilizando un diseño completamente al azar, con cinco repeticiones y dos animales por unidad experimental. Cada unidad experimental se alojó en un corral de mampostería de $3,5 \times 1,2$ $\mathrm{m}$, abierto parcialmente al frente, y equipado con comedero de tres bocas y bebedero de chupón. El periodo de evaluación fue de cuatro semanas.

Experimento 2: Se evaluaron cinco niveles de treonina digestible $(0,42 ; 0,47 ; 0,52$; $0,57$ y $0,62 \%)$, para cerdos en crecimiento. El valor de $0,52 \%$ de treonina digestible es el recomendado por el NRC (1998). Se usaron 50 cerdos ( 25 hembras y 25 machos castrados híbridos; Pietrain $\times$ Landrace $\times$ Yorkshire) distribuidos en cinco tratamientos con cinco repeticiones y dos cerdos (una hembra y un macho castrado) por unidad experimental, con un peso promedio de $24,02 \pm 3,73 \mathrm{~kg}$. Los animales se alojaron en condiciones similares a las del experimento 1. La duración de la fase experimental fue de seis semanas.
En ambos experimentos se proporcionó una dieta a base de sorgo y pasta de soya balanceada con el comando Optimizador (Solver) de Excel. Las dietas fueron isoenergéticas, isoproteicas, isocalcíticas, isofosfóricas, y se utilizó arena para ajustar el volumen y mantener el mismo contenido de todos los nutrientes diferentes a lisina en el experimento 1 y a treonina en el experimento 2 (tablas I, II y III).

Las variables de respuesta para ambos experimentos fueron: comportamiento productivo, dentro del cual se consideró el consumo diario de alimento (CDA), ganancia diaria de peso (GDP), conversión alimenticia (CA), ganancia de carne magra (GCM) y peso final de los cerdos (PF); en las características de la canal se contempló la grasa dorsal inicial y final (GDI y GDF), el porcentaje de carne magra inicial y final (\%CMI y $\% \mathrm{CMF}$ ), el área del músculo longissimus inicial y final (AMLI y AMLF), y la concentración de nitrógeno de la urea plasmática (NUP) al inicio y al final del experimento.

Los animales se pesaron al inicio del experimento; cada semana fue calculado el CDA, GDP y CA. En las características de la canal, la GD y el AML fueron medidos a la altura de la décima costilla, para lo cual se utilizó un equipo de ultrasonido de tiempo real SonoVet 600 marca MEDISON (Medison, Inc., Cipress, California, USA). Los datos de GD, AML, y de peso vivo inicial y final se utilizaron para determinar la GCM y el \%CM con la ecuación del NPPC (1991).

Para determinar la concentración de nitrógeno de la urea plasmática, se tomaron las muestras de sangre mediante punción en la vena cava de los animales, al inicio y al final del experimento, para lo cual se utilizaron tubos vacutainer de $10 \mathrm{ml}$ con heparina (BD Vacutainer, Franklin Lakes, NJ, 07417, USA). Las muestras de sangre obtenidas se identificaron y se conservaron en hielo hasta su centrifugación en el laboratorio. La centrifugación se realizó por diez minutos a $2500 \mathrm{rpm}$ (1286 g), para separar el plasma del paquete celular. El plasma se transfirió a 
Tabla I. Composición de dietas experimentales para el experimento 1. (Composition of experimental diets, experiment 1).

\begin{tabular}{lccccc}
\hline & \multicolumn{5}{c}{ Nivel de lisina digestible en la dieta (\%) } \\
Ingrediente (\%) & 0,67 & 0,75 & 0,83 & 0,91 & 0,99 \\
\hline Pasta de soya & 20,58 & 20,58 & 20,58 & 20,58 & 20,58 \\
Sorgo & 75,92 & 75,92 & 75,92 & 75,92 & 75,92 \\
Aceite & 0,89 & 0,89 & 0,89 & 0,89 & 0,89 \\
Carbonato de calcio & 0,59 & 0,59 & 0,59 & 0,59 & 0,59 \\
Ortofosfato & 0,75 & 0,75 & 0,75 & 0,75 & 0,75 \\
Premezcla de vitaminas* & 0,20 & 0,20 & 0,20 & 0,20 & 0,20 \\
Premezcla de minerales* & 0,10 & 0,10 & 0,10 & 0,10 & 0,10 \\
L-lisina HCL $^{*}$ & 0,10 & 0,20 & 0,30 & 0,41 & 0,51 \\
--treonina $_{\text {DL-metionina }}$ & 0,08 & 0,08 & 0,08 & 0,08 & 0,08 \\
Sal & 0,02 & 0,02 & 0,02 & 0,02 & 0,02 \\
Arena & 0,35 & 0,35 & 0,35 & 0,35 & 0,35 \\
Total & 0,41 & 0,31 & 0,21 & 0,10 & 0,00 \\
& 100,00 & 100,00 & 100,00 & 100,00 & 100,00 \\
\hline
\end{tabular}

*Aportó por kg de alimento: 1,2 UI Vit. E; 80 mg Vit. K; 0,072 mg Vit. $B_{1} ; 200$ mg Vit. $B_{2} ; 1600$ mg Vit. $B_{5}$; $80 \mathrm{mg}$ Vit. $\mathrm{B}_{6} ; 0,0012 \mathrm{mg}$ Vit. $\mathrm{B}_{12} ; 0,004 \mathrm{mg}$ Biotina; $1800 \mathrm{mg}$ Colina; $640 \mathrm{mg}$ Ac. pantoténico; 0,04 mg Ac. fólico; $240 \mathrm{mg}$ antioxidante; $4000 \mathrm{mg} \mathrm{Fe;} 4 \mathrm{mg} \mathrm{Zn}$; y $4 \mathrm{mg} \mathrm{Mg}$.

tubos de polipropileno y se congeló a $-20^{\circ} \mathrm{C}$ por el método colorimétrico propuesto por hasta realizar las determinaciones de urea Searcy et al.(1961).

Tabla II. Composición de dietas experimentales para el experimento 2. (Composition of experimental diets, experiment 2).

\begin{tabular}{lccccc}
\hline & \multicolumn{5}{c}{ Nivel de treonina digestible en la dieta $(\%)$} \\
Ingrediente (\%) & 0,42 & 0,47 & 0,52 & 0,57 & 0,62 \\
\hline Pasta de soya & 17,77 & 17,77 & 17,77 & 17,77 & 17,77 \\
Sorgo & 78,92 & 78,92 & 78,92 & 78,92 & 78,92 \\
Aceite & 0,80 & 0,80 & 0,80 & 0,80 & 0,80 \\
Carbonato de calcio & 0,63 & 0,63 & 0,63 & 0,63 & 0,63 \\
Fosfato & 0,78 & 0,78 & 0,78 & 0,78 & 0,78 \\
Premezcla de vitaminas & 0,20 & 0,20 & 0,20 & 0,20 & 0,20 \\
Premezcla de minerales & 0,10 & 0,10 & 0,10 & 0,10 & 0,10 \\
L-lisina HCL & 0,29 & 0,29 & 0,29 & 0,29 & 0,29 \\
L-treonina & 0,02 & 0,07 & 0,12 & 0,17 & 0,22 \\
DL-metionina & 0,04 & 0,04 & 0,04 & 0,04 & 0,04 \\
L-triptofano & 0,02 & 0,02 & 0,02 & 0,02 & 0,02 \\
Sal & 0,35 & 0,35 & 0,35 & 0,35 & 0,35 \\
Arena & 0,20 & 0,15 & 0,10 & 0,05 & 0,00 \\
TOTAL & 100,00 & 100,00 & 100,00 & 100,00 & 100,00 \\
\hline
\end{tabular}

Aportó por kg de alimento: 1,2 UI Vit. E; 80 mg Vit. K; 0,072 mg Vit. $B_{1} ; 200$ mg Vit. $B_{2} ; 1600$ mg Vit. $B_{5}$; $80 \mathrm{mg}$ Vit. $\mathrm{B}_{6} ; 0,0012 \mathrm{mg}$ Vit. $\mathrm{B}_{12} ; 0,004 \mathrm{mg}$ Biotina; $1800 \mathrm{mg}$ Colina; $640 \mathrm{mg}$ Ac. pantoténico; $0,04 \mathrm{mg}$ Ac. fólico; $240 \mathrm{mg}$ antioxidante; $4000 \mathrm{mg} \mathrm{Fe;} 4$ mg, Zn; 4 mg Mg.

Archivos de zootecnia vol. 59, núm. 226, p. 208. 
Tabla III. Contenido nutrimental de las dietas basales en los experimentos 1 y 2 . (Nutritional concentration of basal diets, experiments 1 and 2).

\begin{tabular}{lcc}
\hline Nutrimento (\%) & $\begin{array}{c}0,67 \% \\
\text { Lisina }\end{array}$ & $\begin{array}{c}0,42 \% \\
\text { Treonina }\end{array}$ \\
\hline EM (Kcal) & 3265 & 3265 \\
Proteína cruda & 16 & 15 \\
Lisina digestible & 0,67 & 0,75 \\
Arginina digestible & 0,85 & 0,77 \\
Treonina digestible & 0,67 & 0,42 \\
Triptófano digestible & 0,20 & 0,16 \\
Metionina + Cistina digestible & 0,54 & 0,47 \\
Isoleucina digestible & 0,60 & 0,55 \\
Valina digestible & 0,67 & 0,63 \\
Leucina digestible & 1,32 & 1,26 \\
Calcio & 0,60 & 0,60 \\
Fósforo total & 0,51 & 0,51 \\
Fósforo digestible & 0,23 & 0,23 \\
\hline
\end{tabular}

Las variables CDA, GDP y CA de los experimentos fueron analizadas utilizando un diseño completamente al azar mediante el procedimiento MIXED (SAS, 2001), y con el procedimiento GLM (General Linear Models; Modelos Lineales Generalizados; SAS, 2001) para las variables evaluadas individualmente al inicio y final del experimento (PI, PF, GDP, GCM, \%CMI, \%CMF, AMLI, AMLF, NUP). Los datos del experimento 2 se analizaron de manera global y por sexo utilizando el procedimiento GLM. La comparación de medias se realizó utilizando la prueba de Tukey (SAS, 2001); mientras que el efecto del nivel de lisina y treonina digestible se evaluó con contrastes ortogonales para detectar tendencias lineales cuadráticas o cúbicas (Steel et al., 1997). En todos los análisis estadísticos se utilizó el peso inicial (PI) como covariable.

\section{RESULTADOS}

\section{EXPERIMENTO 1}

Los resultados de respuesta productiva (tabla IV) mostraron que el PI afectó $(\mathrm{p} \leq 0,01)$ las variables CDA, GDP y PF. Aún cuando no se observó efecto de tratamiento para CDA y GDP, la comparación de medias con Tukey mostró diferencias entre tratamientos para CDA, GDP y CA. El nivel de lisina digestible afectó de manera cúbica el CDA ( $\mathrm{p} \leq 0,05)$, siendo la dieta con $0,75 \%$ de lisina la que presentó el mayor consumo $(\mathrm{p} \leq 0,05)$. La GDP presentó una tendencia $(\mathrm{p} \leq 0,07)$ también cúbica; las dietas con 0,75 y $0,99 \%$ de lisina tuvieron los mayores valores $(\mathrm{p} \leq 0,05)$. Para la CA se observó un efecto lineal $(p \leq 0,01)$, siendo los cerdos alimentados con $0,91 \%$ de lisina los que presentaron la mejor conversión $(p \leq 0,05)$. En cuanto a las características de la canal y el NUP (tabla IV), el PI afectó ( $p \leq 0,01)$ sólo al AMLF. El nivel de lisina digestible afectó cuadráticamente $(\mathrm{p} \leq 0,05)$ el AMLF, el \%CMF y el NUP, pero la comparación de medias con Tukey no mostró diferencias entre tratamientos $(\mathrm{p}>0,05)$ para estas variables.

El factor tiempo (semana) afectó $(p \leq 0,01)$ a las variables CDA, GDP y CA. También se encontró efecto $(p \leq 0,05)$ de la interacción tratamiento por semana de medición en la $\operatorname{GDP}(\mathrm{p} \leq 0,03)$ y CA $(\mathrm{p} \leq 0,01)$, lo que implica un diferente comportamiento de esas variables para cada semana.

\section{EXPERIMENTO2}

Las variables evaluadas en el experimento de treonina (tabla $\mathbf{V}$ ) no fueron afectadas $(p>0,05)$ por el nivel de treonina digestible en la dieta; sólo presentaron un efecto de semana $(\mathrm{p} \leq 0,01)$ y una interacción entre semana y tratamiento $(p \leq 0,01)$. El NUP presentó un efecto lineal $(\mathrm{p} \leq 0,05)$ disminuyendo cuando se incrementó el nivel de treonina en la dieta; sin embargo, no se detectaron diferencias entre tratamientos al comparar las medias con Tukey $(\mathrm{p}>0,05)$. Las variables evaluadas por sexo (PI, PF, GDP, GCM, GDF, AMLF y \%CMF, tabla VI) no mostraron efecto $(p>0,05)$ del nivel de treonina digestible en la dieta, tanto en hembras como en machos castrados. Sólo el NUP mostró un efecto lineal $(\mathrm{p} \leq 0,05)$ en cerdos machos castrados, observándose 
LÓPEZ, FIGUEROA, GONZÁLEZ, MIRANDA, ZAMORA Y CORDERO

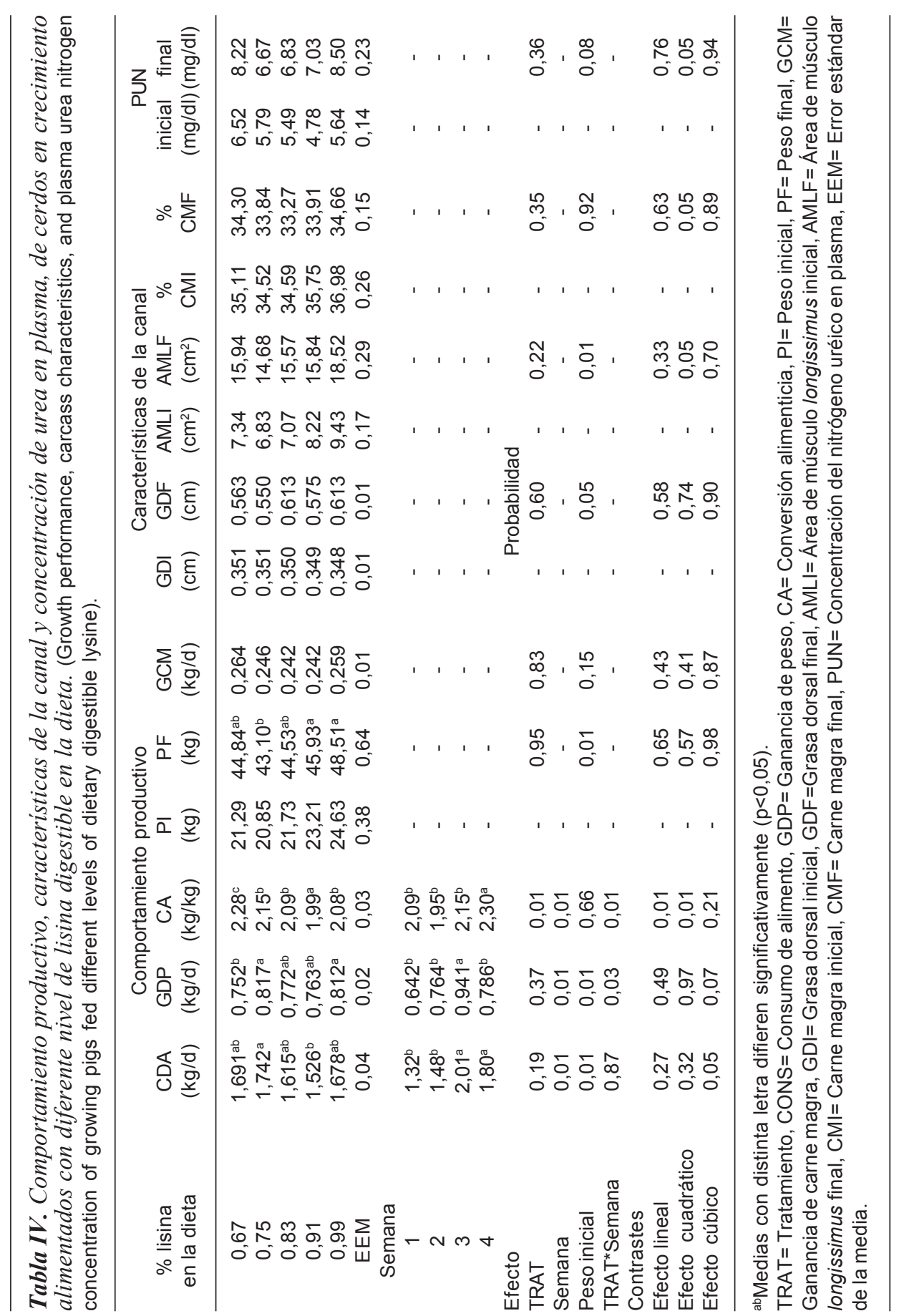

Archivos de zootecnia vol. 59, núm. 226, p. 210. 


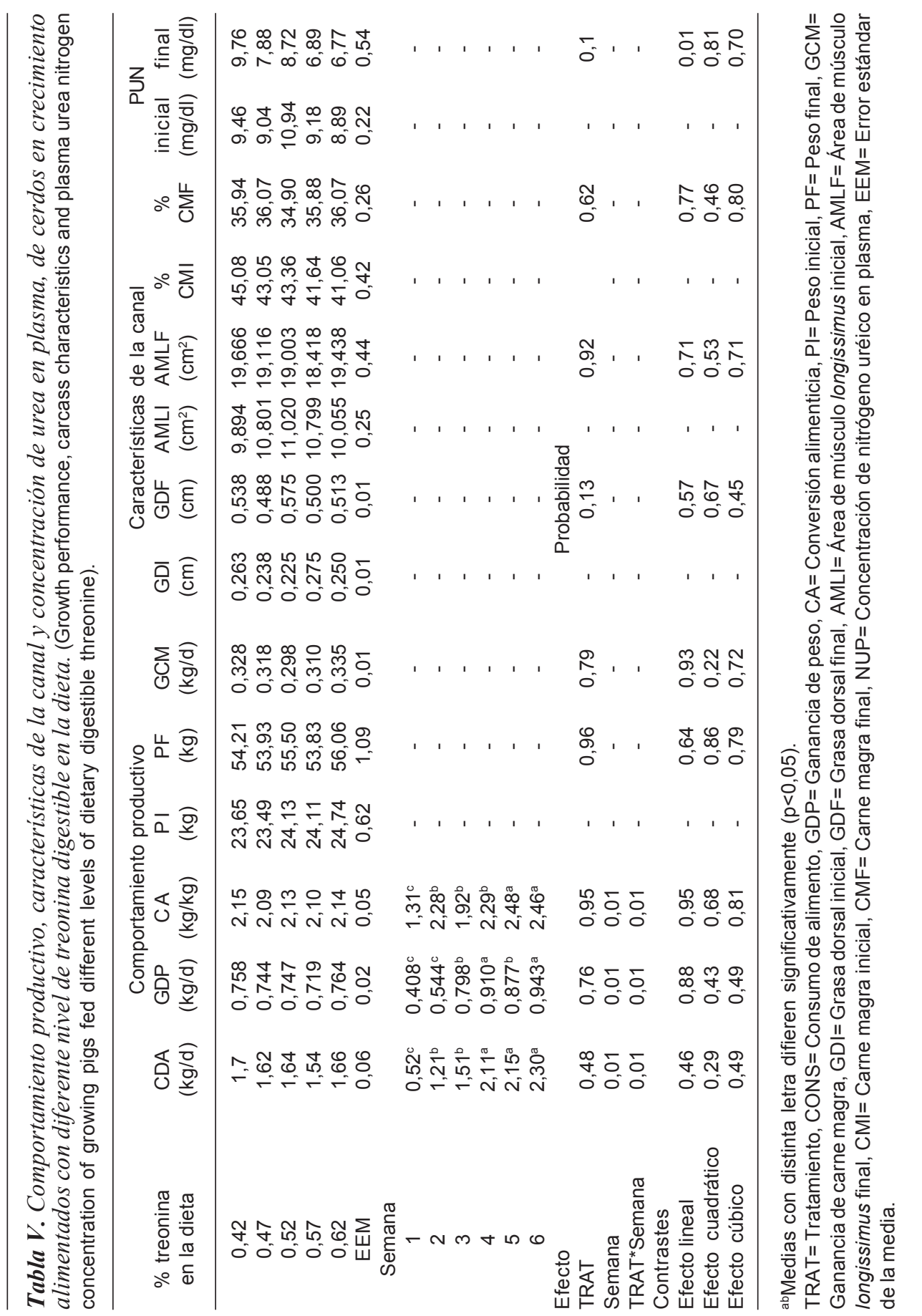




\section{LÓPEZ, FIGUEROA, GONZÁLEZ, MIRANDA, ZAMORA Y CORDERO}

que disminuyó a medida que se incrementó el nivel de treonina en la dieta. El NUP final para hembras no mostró efecto $(\mathrm{p}>0,05)$ del nivel de treonina en la dieta. Al realizar la comparación de medias no se encontraron diferencias entre tratamientos para machos $y$ hembras $(p>0,05)$.

\section{DISCUSIÓN}

\section{EXPERIMENTO1}

Las variables CDA, GDP, CA y PF fueron afectadas por el nivel de lisina en la dieta. La respuesta del CDA coincide con otros estudios que también encontraron efecto del nivel de lisina en la dieta sobre esta variable (Loughmiller et al., 1998), utilizando dietas con base a sorgo-pasta de soya. Sin embargo, Witte et al. (2000), que utilizaron maízpasta de soya, no encontraron efecto del nivel de lisina sobre el consumo de alimento en cerdos en crecimiento y finalización, atribuyéndolo a que las dietas no presentaron deficiencias o excesos de este AA. La eficiencia de utilización de los AA depende de su equilibrio en la dieta, alterando con ello la CA (Sussenbeth, 1995). La GDP coincide con lo encontrado por Pérez et al. (2005), con maíz-pasta de soya, quienes observaron efecto del nivel de lisina sobre esta variable. El efecto que presentó la CA coincide con los resultados de Cline et al. (2000), que también utilizaron maíz-pasta de soya en las dietas. La respuesta en la GCM fue similar en todos los tratamientos, lo que coincide con los datos de Medina (2002), obtenidos en las mismas condiciones (ingredientes, instalaciones y animales) que el presente estudio, donde la GCM no presentó diferencias significativas $(p>0,05)$. De acuerdo con Fuller et al. (1989), el requerimiento de lisina para el cerdo, con respecto al de los demás AA esenciales, es mayor para depositar proteína que el necesario para mantenimiento; probablemente en este estudio los niveles usados fueron menores con respecto a los requerimientos y no fue suficiente para depositar más proteína en la canal. El hecho de que no se observara efecto del nivel de lisina digestible en la dieta sobre la GCM, posiblemente se deba al alto valor de PC en la dieta, que puede estar limitando el efecto de lisina por un exceso de PC, y por lo tanto hay un gasto de energía para eliminar el exceso de $\mathrm{N}$, en lugar de destinarlo a la síntesis de tejido magro (Cline et al., 2000). El NRC (1998) señala que cerdos con GCM de 300, 325 y $350 \mathrm{~g} \mathrm{~d}^{-1}$ son considerados como genotipos de media, media-alta y alta calidad genética, respectivamente. Sin embargo, Stahly et al. (1991) mencionan que cerdos con ganancia de carne magra de 230,230 a $330 \mathrm{y}>340 \mathrm{~g} \mathrm{~d}^{-1}$ son considerados como de baja, media y alta calidad genética, respectivamente. Con base en lo anterior se puede señalar que los cerdos utilizados en esta investigación son de calidad genética media-alta, ya que la GCM va de 298 a $335 \mathrm{~g} \mathrm{~d}^{-1}$, lo que puede explicar que los requerimientos de AA sean mayores para GCM en este tipo de cerdos.

En cuanto a las características de la canal, la selección genética consiguió disminuir la cantidad de grasa en la canal, obteniéndose así cerdos magros, por lo que una mayor cantidad de energía se destina a la síntesis de proteína (Cline et al., 2000). La respuesta no significativa sobre la GDF en el presente trabajo, probablemente se deba a que los animales provienen de razas con un potencial genético medio-alto, con baja acumulación de GD, o bien a un desequilibrio entre AA. Los animales que consumieron dietas con los niveles más altos de lisina digestible, presentaron los valores numéricos más altos de GD, resultados que coinciden con los de Cline et al. (2000), quienes observaron que los niveles más altos de lisina en la dieta $(1,15$ y $1,40 \%)$ provocaron mayor depósito de grasa en los cerdos.

El efecto del PI sobre el AML se atribuye a que los animales más pesados presentan mayor área del músculo longissimus (AMLI) al inicio del experimento, que conlleva a una respuesta similar al final del mismo. El efecto cuadrático $(p<0,05)$ observado en el AML, 


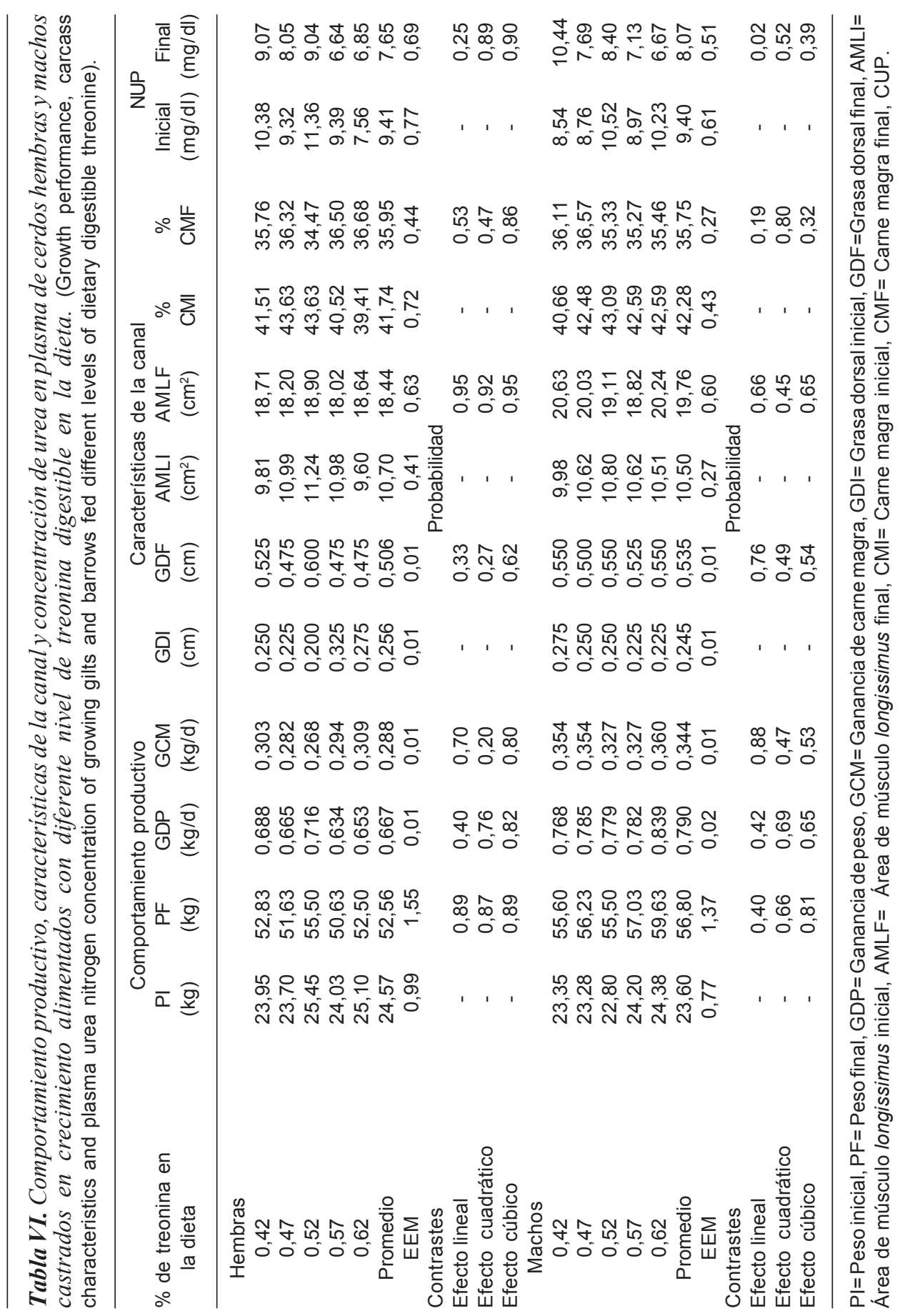




\section{LÓPEZ, FIGUEROA, GONZÁLEZ, MIRANDA, ZAMORA Y CORDERO}

coincide con el reportado en otras investigaciones en cerdos en crecimiento (Hansen y Lewis, 1993; Witte et al., 2000; Medina, 2002). La eficiencia de utilización de lisina y otros AA depende del equilibrio entre ellos, y además, el requerimiento es diferente para cada variable de respuesta. La respuesta del AMLF también se reflejó en el \%CMF en la canal, ya que también mostró un efecto cuadrático del nivel de lisina digestible.

En el NUP se encontró respuesta cuadrática $(\mathrm{p} \leq 0,05)$ disminuyendo al aumentar la lisina digestible, lo que coincide con resultados de Abreu et al. (2007), quienes obtuvieron una respuesta similar al adicionar niveles de lisina digestible muy cerca$\operatorname{nos}(0,70 ; 0,80 ; 0,90, y 1,00 \%)$ a los utilizados en este trabajo. El NUP más alto se observó con los niveles extremos $(0,67 \%$ y $0,99 \%$ ); en el primer caso pudo deberse a que hay un déficit de AA y no alcanzaron el balance adecuado para ser utilizados, por lo que son excretados; y en el segundo caso, posiblemente a que hubo un exceso de AA que igualmente fueron excretados.

\section{EXPERIMENTO2}

Los resultados indicaron que no hubo efecto del nivel de treonina digestible en la dieta sobre el comportamiento productivo y las características de la canal en cerdos en crecimiento (tabla V), lo que pudo deberse a que los niveles de treonina digestible fueron muy cercanos entre sí (con sólo $0,05 \%$ entre tratamientos). Estos resultados no coinciden con los de Ettle et al. (2004), quienes encontraron que la adición de treonina sintética a la dieta afecta la GDP y la CA Sin embargo, los resultados fueron semejantes a Figueroa et al. (1999) en donde no se observó efecto de treonina sobre el comportamiento productivo, lo que supuestamente pudo deberse a que las dietas sorgopasta de soya con $16 \%$ de PC contenía suficiente cantidad tanto de treonina como de los demás AA. Esto mismo pudo ocurrir en el presente experimento, ya que el nivel que $\mathrm{PC}$ fue de $15 \% \mathrm{y}$ se adicionaron AA sintéticos (L-lisina HCl, DL-metionina, Ltriptófano y L-treonina). Hansen et al. (1993) sugieren un mínimo de $14 \%$ de PC en dietas sorgo-pasta de soya, adicionadas con lisina y treonina sintéticos, para obtener una máxima respuesta productiva.

El hecho de que tampoco se observaran efectos del nivel de treonina sobre las características de la canal, concuerda con lo reportado por Donzele (2005). Además, se ha observado que la influencia de la adición de treonina sobre las características de la canal es menor que en las variables de comportamiento productivo (Ettle et al., 2004).

La disminución de NUP al incrementar la treonina en la dieta, ya había sido reportado por Berto et al. (2002) y Wang et al. (2006) en cerdos en iniciación, lo que indica que con niveles bajos de treonina existe un desequilibrio entre AA, lo que impide que estos nutrientes puedan ser aprovechados eficientemente por el cerdo, reflejándose en una mayor concentración de urea en plasma, que posteriormente es excretada en la orina; mientras que con niveles mayores de treonina existe un mejor equilibrio entre AA, lo cual conduce a que sean utilizados con mayor eficiencia por el animal, lo que se deduce por una baja concentración de urea plasmática. Esto indica que se requiere más treonina en la dieta para poder obtener una máxima respuesta productiva y un mejoramiento en las características de la canal.

Los machos castrados (tabla VI) presentaron mayor GDP, GCM y AMLF que las hembras. Este resultado pudo deberse principalmente a la mayor capacidad de consumo de alimento que presentan los machos castrados, y por tanto, mayor disponibilidad de AA para la síntesis de tejido magro. Sin embargo, las hembras tuvieron menor GD en comparación con los machos, resultados que concuerdan con los de Hansen y Lewis (1993); y Friesen et al. (1994). Estas diferencias pueden atribuirse a que las hembras tienen un menor consumo de alimento que los machos y con ello una menor disponibilidad de energía para la síntesis de grasa. 


\section{LISINA Y TREONINA DIGESTIBLE EN DIETAS PARA CERDOS EN CRECIMIENTO}

En el caso del NUP final, los cerdos machos castrados presentaron un efecto lineal ( $\mathrm{p} \leq 0,05)$, disminuyendo la concentración a medida que se incrementó el nivel de treonina en la dieta; este resultado es similar al obtenido en cerdos en iniciación (Wang et al., 2006), y pudiera indicar que los machos castrados tienen mayor requerimiento de treonina en comparación con las hembras; o bien, que las hembras son más eficientes en la utilización de AA (Friesen et al., 1994; Cline et al., 2000) que los machos castrados.

\section{CONCLUSIONES}

El mejor nivel de lisina digestible en dietas para cerdos en crecimiento depende de la variable en estudio: para consumo de

\section{BIBLIOGRAFÍA}

Abreu, M.L.T. de, Donzele, J.L., Miranda, O.R.F de, Oliveira, A.L.S. de, Santos, F. de A. e Pereira, A.A. 2007. Níveis de lisina digestível em rações, utilizando-se o conceito de proteína ideal, para suínos machos castrados de alto potencial genético para deposição de carne magra na carcaça dos 60 aos $95 \mathrm{~kg}$. Rev. Bras. Zootecn., 36: 54-61.

Araki, K. 1990. Production of amino acid. In: Nutrition. Protein and amino acids. Yoshida, $\mathrm{H}$ Naito, Y. Nuyama and T. Susuki (eds.). Japan Sci. Soc. Press. Tokio, Japan.

Berto, A.D., Wechsler, S.F. e Noronha, C.C. 2002. Exigências de treonina de leitões dos 7 aos 12 e dos 12 aos $23 \mathrm{~kg}$. Rev. Bras. Zootecn., 31: 1176-1183.

Cline, T.R., Cromwell, G.L., Crenshaw, T.D., Ewan, R.C., Hamilton, C.R., Lewis, A.J., Mahan, D.C. and Southern, L.L. 2000. Further assessment of the dietary requirements of finishing gilts. $J$. Anim. Sci., 78: 987-992.

Coma, J., Carrion, D. and Zimmerman, R.D. 1995. Use of plasma urea nitrogen as a rapid response criterion to determine the lysine requeriment of pigs. J. Anim. Sci., 73: 472-481.

Donzele, J.L. 2005. Requerimientos de treonina digestible de cerdos machos castrados de alto alimento y ganancia de peso, $0,75 \%$ de lisina digestible, presentó el mayor consumo y la mayor ganancia. Sin embargo, la conversión alimenticia fue mejor con $0,91 \%$. Otras variables en estudio también fueron afectadas: el área del músculo longissimus mejoró al aumentar el nivel de lisina digestible, mientras que el porcentaje de carne magra y la concentración de urea en plasma presentaron los mayores valores con el nivel más bajo y con el más alto de lisina. En cuanto al nivel de treonina digestible en dietas para cerdos en crecimiento, no se pudo determinar cuál es el mejor nivel de este aminoácido, ya que no se observaron diferencias entre tratamientos. El nivel de treonina digestible, en el análisis por sexos, sólo afectó la concentración de urea en plasma de machos castrados.

potencial genético, desde los 95 a los $125 \mathrm{~kg}$ Ajinomoto Animal Nutrition. Informe de investigación, $n^{\circ}$ 43. www.lysine.com. (17-2-2007).

Ettle, T., Roth-Maier, D.A., Bartelt, J. and Roth, F.X. 2004. Requirement of true ileal digestible threonine of growing and finishing pigs. J. Anim. Physiol. An. N., 88: 211-222.

Ferreira, R.A., Miranda, O.R.F. de, Lopes, D.J., Viera, A.C. de, Oliveira, S.F.C. de, Oliveira, F.D. de e Paes, S.E. 2005. Reducto do nível de proteína bruta e suplementação de aminoácidos em rações para suinos machos castrados mantidos em ambiente termoneutro dos 30 aos 60 kg. Rev. Bras. Zootecn., 34: 548:556.

Figueroa, J.L., Cervantes, M. and Cuca, M. 1999. Lysine and threonine sources for growing pigs under heat stress. Cuban J. Agric. Sci., 33: 183-189.

Fontes, O.D. de, Donzele, J.L., Miranda, O.R.F. de, Silva, C.G. e Aragão, P. 2000. Níveis de lisina para leitoas selecionadas geneticamente para deposição de carne magra, dos 30 aos 60 kg, mantendo constante a relação entre lisina e metionia+cistina, treonina, triptofano, isoleucina e valina. R. Bras. Zootecn., 29: 776-783.

Friesen, K.G., Nelssen, J.L., Goodband, R.D., Tokach, M.D., Unruh, J.A., Kropf, D.H. and Kerr, 


\section{LÓPEZ, FIGUEROA, GONZÁLEZ, MIRANDA, ZAMORA Y CORDERO}

B.J. 1994. Influence of dietary lysine on growth and carcass composition of high-lean-growth gilts fed from 34 to 72 kilograms. J. Anim. Sci., 72: $1761-1770$

Fuller, M.F., McWilliams, R., Wang, T.C. and Giles, L.R. 1989. The optimum dietary amino acids pattern for pigs. 2. Requirements for maintenance and for tissue accretion. Br. J. Nutr., 61: 253267.

García, E. 1988. Modificación al sistema de clasificación climática de Koppen para adaptarlo a las condiciones particulares de la República Mexicana. Talleres Offset Larios. México. D.F. $277 \mathrm{pp}$.

Hahn, J.D., Biehl, R.R. and Baker, D.H. 1995. Ideal digestible lysine level for early- and late-finishing swine. J. Anim. Sci., 73: 773-784.

Hansen, B.C. and Lewis, A.J. 1993. Effects of dietary protein concentration (corn: soybean meal ratio) on the performance and carcass characteristics of growing boars, barrows, and gilts: mathematical descriptions. J. Anim. Sci., 71: 2122-2132.

Hansen, J.A., Knabe, D.A. and Burgoon, K.G. 1993. Amino acids supplementation of lowprotein, sorghum-soybean meal diet for 20 to 50-kilogram swine. J. Anim. Sci., 71: 442-451. Loughmiller, J.A., Nelssen, J.L., Goodband, R.D., Tokach, M.D., Titgemeyer, E.C. and Kim, I.H. 1998. Influence of dietary total sulfur amino acids and methionine on growth performance and carcass characteristics of finishing gilts. $J$. Anim. Sci., 76: 2129-2137.

Medina, R. 2002. Optimización biológica y económica de niveles de lisina total en dietas para cerdos en crecimiento. Tesis de Maestría en Producción Animal. Departamento de Zootecnia. Universidad Autónoma Chapingo. 91 pp.

NPPC. 1991. Procedures to evaluate market hogs. $3^{\text {rd }}$ ed. National Pork Producers Council. Des Moines, IA.

NRC. 1998. Nutrient requirements of swine. $10^{\text {th }}$ ed. National Academy Press, Washington, D.C.

Pérez, A., Obispo, N.E., Palma, J. y Chicco, C.F. 2005. Efectos de la ractopamina y el nivel de lisina sobre la respuesta productiva de cerdos magros en la fase de engorda. Rev. Zootec. Trop., 23: 429-445.

Pichardo, A., Cervantes, R.M., Cuca, M., Figueroa, J.L., Araiza, A.B., Torrentera, N. and Cervantes, M. 2003. Limiting amino acids in wheat for growing-finishing pigs. Interciencia, 28: 287291.

Saldana, C.I., Knabe L.M., Owen, K.Q., Burgoon, K.G. and Gregg, E.J. 1994. Digestible threonine requirements of starter and finisher pigs. $J$. Anim. Sci., 72: 144-150.

SAS. 2001. S.A.S. User's guide: Statistics. $8^{\text {th }}$ ed. SAS Institute Inc., Cary, N.C. USA.

Searcy, R.L., Gough, G.S., Korotzer, J.L. and Bergquist, L.M. 1961. Evaluation of a new technique for estimation of urea nitrogen in serum. Am. J. Med. Tech., 27: 255-262.

Steel, D.R.G., Torrie, J.H. and Dickey, D.A. 1997. Principles and Procedures of Statistics. A Biometrical Approach. $3^{\text {rd }}$ ed. McGraw-Hill Book Co. New York.

Stahly, T.S., Cromwell, G.L. and Terhune, D. 1991. Responses of high, medium and low lean growth genotypes to dietary amino acid regimen. $J$. Anim. Sci., 69(Suppl. 1): 364 (Abstract).

Sussenbeth, A. 1995. Factors affecting lysine utilization in growing pigs: an analysis of literature data. Livest. Prod. Sci., 43: 193-204.

Wang, X., Qiao, S.Y., Lui, M. and Ma, Y.M. 2006. Effects of graded levels of true ileal digestible threonine on performance, serum parameters and immune function of $10-25 \mathrm{~kg}$ pigs. Anim. Feed Sci. Tech., 129: 264-278.

Wei, R. and Zimmerman, D.R. 1998. Lysine requirements of PIC barrows during growingfinishing period. Nutrition. Department of Animal Science. lowa State University. pp. 39-41. http:/ /www.ipic.iastate.edu/reports/00swinere ports/asl-653.pdf (18-4-2006).

Witte, D.P., Millis, M., McKeith, F.K. and Wilson, E.R. 2000. Effect of dietary lysine and environmental temperature during the finishing phase on the intramuscular fat content of pork. J. Anim. Sci., 78: 1272-1276.

Archivos de zootecnia vol. 59, núm. 226, p. 216. 